\title{
Cardiac and Cerebral Arterial Complications of Lemierre Syndrome: Results from a Systematic Review and Individual Patient Data Meta-analysis
}

\author{
William Pleming ${ }^{1}$ Stefano Barco ${ }^{1,2}$ Davide Voci ${ }^{1}$ Clara Sacco $^{3}$ Federica Zane ${ }^{4}$ Serena Granziera ${ }^{5}$ \\ Gabriele Corsi ${ }^{6,7}$ Stavros V. Konstantinides ${ }^{2}$ Nils Kucher ${ }^{1}$ Alessandro Pecci ${ }^{8} \quad$ Luca Valerio $^{20}$ \\ ${ }^{1}$ Clinic of Angiology, University Hospital Zurich, Zurich, Switzerland \\ Address for correspondence Luca Valerio, MD, Center for Thrombosis \\ ${ }^{2}$ Center for Thrombosis and Hemostasis, Mainz University Medical \\ and Hemostasis, Mainz University Medical Center, Langenbeckstrasse \\ Center, Mainz, Germany \\ 1, 55131 Mainz, Germany (e-mail: luca.valerio@uni-mainz.de).
}

${ }^{3}$ Center for Thrombosis and Hemorrhagic Diseases, IRCCS Humanitas

Research Hospital, Rozzano, Milan, Italy

${ }^{4}$ Department of General Medicine, Hospital of Sondrio, Sondrio, Italy

${ }^{5}$ Department of Medicine, Geriatric Unit, Ospedale San Giovanni e Paolo, Venice, Italy

${ }^{6}$ Department of Clinical, Integrated and Experimental Medicine (DIMES), Respiratory and Critical Care Unit, S. Orsola - Malpighi Hospital, Alma Mater University, Bologna, Italy

${ }^{7}$ IRCCS Azienda Ospedaliero - Universitaria of Bologna, Bologna, Italy

${ }^{8}$ Department of Internal Medicine, IRCCS Policlinico San Matteo

Foundation and University of Pavia, Pavia, Italy

Hamostaseologie 2022;42:261-267.

\section{Abstract}

Keywords

- Lemierre syndrome

- thrombosis

- stroke

- septic embolism

- anticoagulation

- Fusobacterium necrophorum
Background Lemierre syndrome is a potentially life-threatening disease, which affects otherwise healthy young adults and adolescents. It is characterized by acute neck vein thrombosis and septic embolism, usually complicating a bacterial infection. Data on the syndrome are sparse, particularly concerning arterial complications.

Methods We evaluated the frequency and patterns of cerebral arterial and cardiac involvement ("arterial complications") in an individual patient level cohort of 712 patients, representing all cases described over the past 20 years in the medical literature who fulfilled the criteria: (1) bacterial infection in the neck/head site and (2) objectively confirmed thrombotic complication or septic embolism. The study outcomes were defined as all-cause in-hospital deaths and the occurrence of clinical sequelae at discharge or in the postdischarge period.

Results A total of 55 (7.7\%) patients had an arterial complication. The most frequent arterial complications were carotid involvement (52.7\%), stroke (38.2\%), and pericardial complications (20\%). Patients with an arterial involvement were more likely to be treated with a greater number of antibiotics ( 23 vs. $10 \%$ ) and to receive anticoagulation. In addition, patients with arterial complications had a greater risk of all-cause death $(n=20 / 600,3.3 \%$ vs. $n=6 / 52,12 \%$; odds ratio [OR]: 3.8 ; 95\% confidence interval [Cl]: $1.5-9.9)$ and late clinical sequelae ( $n=49 / 580,9.0 \%$ vs. $n=15 / 46,35 \%$; OR: 5.2 ; $95 \%$ Cl: $2.65-10.37$ ). received

June 18, 2021

accepted after revision

November 10, 2021
DOI https://doi.org/

10.1055/a-1694-8723.

ISSN 0720-9355. (c) 2022. The Author(s).

This is an open access article published by Thieme under the terms of the Creative Commons Attribution-NonDerivative-NonCommercial-License, permitting copying and reproduction so long as the original work is given appropriate credit. Contents may not be used for commercial purposes, or adapted, remixed, transformed or built upon. (https://creativecommons.org/ licenses/by-nc-nd/4.0/)

Georg Thieme Verlag KG, Rüdigerstraße 14, 70469 Stuttgart,

Germany 
Conclusions While Lemierre syndrome is known to be primarily characterized by venous thromboembolic events, our results suggest that local or distant arterial complications may occur in approximately one-tenth of patients and may be associated with a greater risk of long-term sequelae and death.

\section{Introduction}

Lemierre syndrome is a potentially life-threatening disease which primarily affects otherwise healthy, young individuals. It is characterized by an acute head and neck bacterial infection, often tonsillitis, leading to local vein thrombosis, septic embolism, abscess formation and/or generalized sepsis. ${ }^{1,2}$ The most commonly isolated pathogen is Fusobacterium necrophorum, a gram-negative anaerobe often found colonizing the oropharynx of healthy individuals., ${ }^{3,4}$ Given the eponym after André Lemierre who described the condition in 1936, this syndrome is still poorly understood due to the scarceness of systematic research.

Although once thought of as a forgotten disease, its incidence has been recently perceived as increasing, which has been tentatively attributed to decreased broad-spectrum antibiotic use for head and neck infections in children and young adults. ${ }^{5,6}$ In the last century, complex management decisions, particularly regarding thromboembolic manifestations and more recently anticoagulation, have been made based on a limited selection of available research and case studies. Venous thrombotic complications have been considered typical of the syndrome with little emphasis placed on arterial involvement.

The characteristic local venous thrombosis (most often affecting the internal jugular vein) is known to have wideranging clinical manifestations with frequent occurrence of septic emboli to the lungs. Intracranial venous thrombotic extension is also a common and serious finding as well as septic emboli to the joints and organs such as the liver and spleen. ${ }^{1,2}$ Although the venous thromboembolic phenomena are better known and have been more extensively documented, arterial and cardiac complications of Lemierre syndrome have also been reported. These range from intracranial infarcts to carotid involvement, such as thrombosis, stenosis, or mycotic aneurysm, as well as cardiac complications, such as pericardial disease, infective endocarditis, or cardiac thrombi. ${ }^{7-11}$

More detailed knowledge of the frequency and patterns of cerebral arterial and cardiac involvement would enable us to tailor our treatments accordingly and modify our diagnostic approach. Owing to the lack of information on this topic, we performed an individual patient level analysis of 712 patients with Lemierre syndrome to describe the frequency and patterns of arterial and cardiac involvement.

\section{Methods}

Our methods were adapted from previous studies investigating rare conditions based on case reports on case series and with similar aims, ${ }^{12-14}$ and have been previously de- scribed in detail. ${ }^{2,15}$ In brief, literature reviews were performed for the period January 2000 to August 2017 to identify all studies with relevant cases. Cases were considered to be eligible based on previously proposed definitions of Lemierre syndrome. Cases described before the year 2000 were not included to minimize bias potentially introduced by heterogeneity in patient treatment, as this is the approximate time when low-molecular-weight heparins were introduced worldwide for parenteral anticoagulation. ${ }^{15}$ MEDLINE (via PubMed), Embase (via Ovid), the Cochrane Library, and the gray literature (other relevant results not available through traditional academic publication means) were searched along with the references of all relevant articles. There were no restrictions on language or study design. Titles and abstracts were reviewed by three reviewers who assessed the full texts for eligibility. In cases of disagreement, agreement was reached by consensus. To reduce the risk of publication bias, the authors of single studies were contacted and asked whether they had treated patients with Lemierre syndrome before or since the case or cases described in their case report or case series.

This strategy led to retrieval of 540 studies. Of these, 480 were single case reports and 60 were case series, including 48 series describing up to 5 cases, 16 series of 6 to 20 cases, and 2 series of more than 20 cases $(N=22$ and $N=33)$. Of these studies, complete individual patient-level data were available for 712 patients.

The data collected included year of diagnosis, age in years at the time of diagnosis, sex, isolation of Fusobacterium spp. or other bacterial genera from biological material, evidence of jugular vein thrombosis at the time of presentation, evidence of thrombotic involvement in other venous districts both at presentation and during clinical course, antibiotics used during the clinical course (if any), diagnostic delay (reported time from preclinical symptom onset to diagnosis of Lemierre syndrome in days or weeks), length of hospitalization, and clinical events occurring after the diagnosis of Lemierre syndrome, including new or recurring venous thrombosis, new peripheral septic embolization, bleeding, surgery, death, as well as functional and neurological sequelae still present at the time of discharge among survivors.

In our study, we primarily aimed to provide current estimates of the arterial and cardiac complications among patients diagnosed with Lemierre syndrome. Cerebral or cardiac arterial complications have been defined as the following:

- Any cerebral arterial event: stroke in a cerebral arterial territory, any carotid involvement (e.g., diagnosed thrombosis as such non-otherwise specified "stenosis" or mycotic aneurysm). 
Table 1 Sample characteristics, overall and by presence of any arterial/cardiac complication at any time of the clinical course

\begin{tabular}{|c|c|c|c|}
\hline & $\begin{array}{l}\text { Overall } \\
(N=712)\end{array}$ & $\begin{array}{l}\text { No arterial involvement } \\
(N=657)\end{array}$ & $\begin{array}{l}\text { Arterial involvement } \\
(N=55)\end{array}$ \\
\hline Typical Lemierre syndrome & $333(47 \%)$ & $315(48 \%)$ & $18(33 \%)$ \\
\hline Age (years), median (IQR) & $21(17,33)$ & $21(17,33)$ & $22(16,28)$ \\
\hline Cancer & $12(1.7 \%)$ & $12(1.8 \%)$ & $0(0 \%)$ \\
\hline Any previous medical contact before presentation & $286(40 \%)$ & $268(41 \%)$ & $18(33 \%)$ \\
\hline \multicolumn{4}{|l|}{ Primary infection } \\
\hline Oropharyngeal infection & $520(73 \%)$ & $486(74 \%)$ & $34(62 \%)$ \\
\hline Lower respiratory tract infection & $330(46 \%)$ & $325(49 \%)$ & $5(9.1 \%)$ \\
\hline Neck infection & $287(40 \%)$ & $272(41 \%)$ & $15(27 \%)$ \\
\hline Other infection & $165(23 \%)$ & $144(22 \%)$ & $21(38 \%)$ \\
\hline Any bacterial isolation & $583(82 \%)$ & $534(81 \%)$ & $49(89 \%)$ \\
\hline Gram-positive & $180(25 \%)$ & $160(24 \%)$ & $20(36 \%)$ \\
\hline Gram-negative & $474(67 \%)$ & $441(67 \%)$ & $33(60 \%)$ \\
\hline Fusobacterium spp. & $415(58 \%)$ & $387(59 \%)$ & $28(51 \%)$ \\
\hline Venous thrombosis of head or neck at diagnosis & $596(84 \%)$ & $545(83 \%)$ & $51(93 \%)$ \\
\hline Thrombosis of internal jugular vein at diagnosis & $526(74 \%)$ & $487(74 \%)$ & $39(71 \%)$ \\
\hline Cerebral vein thrombosis at diagnosis & $143(20 \%)$ & $114(17 \%)$ & $29(53 \%)$ \\
\hline Thrombosis of external jugular vein at diagnosis & $43(6.0 \%)$ & $37(5.6 \%)$ & $6(11 \%)$ \\
\hline Other head-neck vein thrombosis at diagnosis & $116(16 \%)$ & $99(15 \%)$ & $17(31 \%)$ \\
\hline Venous thrombosis outside head, neck, or arm at diagnosis & $15(2.1 \%)$ & $14(2.1 \%)$ & $1(1.8 \%)$ \\
\hline Septic emboli at diagnosis & $582(82 \%)$ & $531(81 \%)$ & $51(93 \%)$ \\
\hline Pulmonary septic emboli at diagnosis & $506(71 \%)$ & $471(72 \%)$ & $35(64 \%)$ \\
\hline Cerebral septic emboli at diagnosis & $79(11 \%)$ & $52(7.9 \%)$ & $27(49 \%)$ \\
\hline Musculoskeletal septic emboli at diagnosis & $108(15 \%)$ & $91(14 \%)$ & $17(31 \%)$ \\
\hline Hepatic septic emboli at diagnosis & $14(2.0 \%)$ & $13(2.0 \%)$ & $1(1.8 \%)$ \\
\hline Other septic embolism at diagnosis & $50(7.0 \%)$ & $34(5.2 \%)$ & $16(29 \%)$ \\
\hline
\end{tabular}

Note: Typical Lemierre syndrome: oropharyngeal infection and isolation of Fusobacterium spp.

- Any cardiac event: either pericardial (further classified as pericardial abscess/hemorrhage or nonabscess, nonhemorrhagic pericardial effusion-transudate or exudate) or nonpericardial cardiac (further classified as cardiac thrombus or infective endocarditis).

For simplicity, arterial and cardiac complications can hereafter be referred to as simply arterial complications. An extensive description of cerebral venous events and other septic embolic events has been provided in the primary analysis of this cohort. ${ }^{2,15}$ The time points of interest during the clinical course were present on clinical presentation (date of hospitalization or diagnosis) or postdiagnosis clinical course.

We described the in-hospital fatality rate as well as the reported rate of long-term complications. Long-term complications were classified as (1) clinical sequelae including cranial nerve palsy or other long-term neurologic defects, orthopaedic, or other functional limitations; (2) new septic thromboembolism or a novel diagnosis of Lemierre syn- drome after discharge; and (3) need for admission to a rehabilitation institution.

For this article, we performed a descriptive analysis of patients with and without arterial complications. Lacking a clinical or statistical ground to do otherwise, we abstained from conducting inferential analysis to compare these two groups. The in-hospital fatality rate and that of long-term sequelae were compared by odds ratios (ORs) and corresponding 95\% confidence intervals (CIs).

\section{Results}

Of the 712 patients in the entire cohort, 55 (7.7\%) had an arterial complication. Of these, 8 were described in the period 2001-2005, 20 in 2006-2010, and 27 in 20112016. Information was available only for baseline (at presentation) in 3 patients, until death or discharge from hospital in 28 patients, and beyond discharge in 24 patients. - Table 1 shows the sample characteristics in the overall population and in patients with or without any arterial complication at 
Table 2 Distribution of arterial complications at any time of the clinical course

\begin{tabular}{|c|l|}
\hline & $\begin{array}{l}\boldsymbol{N}=\mathbf{5 5} \\
\boldsymbol{n}(\%)\end{array}$ \\
\hline Any arterial or cardiac involvement & $55(100 \%)$ \\
\hline Arterial, noncardiac involvement & $40(72.7 \%)$ \\
\hline Stroke & $21(38.2 \%)$ \\
\hline Carotid involvement & $29(52.7 \%)$ \\
\hline Thrombosis & $10(18.2 \%)$ \\
\hline Stenosis & $19(34.5 \%)$ \\
\hline Mycotic aneurysm & $5(9.1 \%)$ \\
\hline Other arterial involvement & $2(3.6 \%)$ \\
\hline Cardiac involvement & $16(29.1 \%)$ \\
\hline Pericardial & $11(20 \%)$ \\
\hline Pericardial abscess or hemorrhage & $6(10.9 \%)$ \\
\hline Pericardial effusion (non-abscess, & $5(9.1 \%)$ \\
\hline non-hemorrhagic. transudate/exudate) & \\
\hline Nonpericardial & $6(10.9 \%)$ \\
\hline Infective endocarditis & $5(9.1 \%)$ \\
\hline Cardiac thrombus & $2(3.6 \%)$ \\
\hline
\end{tabular}

baseline or any time in the postdiagnosis phase. Those with and without arterial involvement had similar age (median: 22 [Q1-Q3 16, 28] vs. 21 [Q1-Q3 17, 33], respectively) and sex prevalence. Although the prevalence of a primary oropharyngeal infection seemed similar in both cohorts $(n=34$, $62 \%$ vs. $n=486,74 \%$ ), a lower respiratory tract infection as a primary source appeared less likely in the group with arterial involvement ( $n=5,9.1 \%$ vs. $n=325,49 \%$ ). Data regarding head and neck vein involvement other than the internal jugular vein suggest that patients with arterial complications more likely had advanced local venous thrombotic disease, including cerebral vein thrombosis at diagnosis $(n=29,53 \%$ vs. $n=114,17 \%)$ and other head-neck vein thrombosis $(n=17$, $31 \%$ vs $n=99,15 \%)$. Moreover, they more frequently presented cerebral septic emboli ( $n=27,49 \%$ vs. $n=52,7.9 \%$ ) and nonpulmonary, noncerebral septic emboli ( $n=16,29 \%$ vs $\mathrm{n}=34,5.2 \%$ ).

The distribution of arterial complications is shown in -Table 2. The most frequent arterial complications were carotid involvement (52.7\%) and stroke (38.2\%). Cardiac complications were reported in $29 \%$ of cases, being more frequently represented by pericardial complications (20\%).

Treatment patterns and clinical outcomes were evaluated in 652 patients with complete postdiagnosis follow-up, consistent with what has been done in the primary analysis. ${ }^{2}$ Antibiotic regimens were similar between the two groups, although a greater number of different antibiotics were likely to be used in patients with arterial involvement (five or more antibiotics used during clinical course, $n=12,23 \%$ vs. $n=60$, 10\%; - Table 3). Patients with arterial involvement were slightly more likely to receive anticoagulation $(n=35,67 \%$ vs. $n=327,55 \%$ ) as well as to undergo surgical procedures $(n=38,73 \%$ vs. $n=299,50 \%)$.

Table 3 Treatment patterns in patients with and without arterial involvement during postdiagnosis clinical course

\begin{tabular}{|c|c|c|}
\hline & $\begin{array}{l}\text { No arterial involvement } \\
N=600\end{array}$ & $\begin{array}{l}\text { Arterial involvement } \\
N=52\end{array}$ \\
\hline Antibiotic therapy during clinical course, $n$ (\%) & $594(99 \%)$ & $52(100 \%)$ \\
\hline Penicillins & $333(56 \%)$ & $24(46 \%)$ \\
\hline Metronidazole & $282(47 \%)$ & $29(56 \%)$ \\
\hline Cephalosporins & $254(42 \%)$ & $21(40 \%)$ \\
\hline Other antibiotic therapy & $397(61 \%)$ & $37(71 \%)$ \\
\hline Five or more antibiotics used during clinical course & $60(10 \%)$ & $12(23 \%)$ \\
\hline Any cardiac (pericardial and nonpericardial) involvement & - & $7(1.1 \%)$ \\
\hline Anticoagulation during clinical course & $327(55 \%)$ & $35(67 \%)$ \\
\hline Low-molecular-weight heparins & $209(35 \%)$ & $20(38 \%)$ \\
\hline Unfractionated heparin & $63(10 \%)$ & $13(25 \%)$ \\
\hline Fondaparinux & $6(1.0 \%)$ & $0(0 \%)$ \\
\hline Direct oral anticoagulants & $3(0.5 \%)$ & $0(0 \%)$ \\
\hline Switch to vitamin $\mathrm{K}$ antagonists & $122(20 \%)$ & $9(17 \%)$ \\
\hline Surgical procedures during clinical course & $299(50 \%)$ & $38(73 \%)$ \\
\hline Abscess drainage & $217(36 \%)$ & $28(54 \%)$ \\
\hline Mastoidectomy & $34(6 \%)$ & $6(12 \%)$ \\
\hline Jugular vein ligation with or without thrombectomy & $26(4 \%)$ & $5(10 \%)$ \\
\hline Other surgery & $94(16 \%)$ & $20(39 \%)$ \\
\hline
\end{tabular}


Table 4 Clinical outcomes

\begin{tabular}{|l|l|l|l|}
\hline & $\begin{array}{l}\text { No arterial } \\
\text { involvement }\end{array}$ & $\begin{array}{l}\text { Arterial } \\
\text { involvement }\end{array}$ & $\begin{array}{l}\text { Odds ratio (95\% Cl) } \\
\text { for arterial } \\
\text { involvement vs none }\end{array}$ \\
\hline Death, $n / N(\%)$ & $20 / 600(3.3 \%)$ & $6 / 52(12 \%)$ & $3.8(1.5-9.9)$ \\
\hline Sequelae at discharge among survivors, $n / N(\%)$ & $49 / 580(9.0 \%)$ & $15 / 46(35 \%)$ & $5.2(2.65-10.37)$ \\
\hline Discharge to a rehabilitation or long-term institution, $n / N(\%)$ & $6 / 580(1.1 \%)$ & $6 / 46(14 \%)$ & $14.4(4.4-46.5)$ \\
\hline Postdischarge recurrence of Lemierre syndrome, $n / N$ & $2 / 580(0.3 \%)$ & $1 / 46(2.1 \%)$ & $6.4(0.6-72.2)$ \\
\hline
\end{tabular}

Note: Death is defined among the 652 patients with complete clinical course, sequelae among the 626 survivors.

-Table 4 shows the clinical outcomes with all-cause death defined among the 652 patients with a complete course reported and clinical sequelae at discharge or after discharge calculated among the 626 survivors. Those with arterial or cardiac involvement were at a greater risk of allcause death ( $n=6 / 52,12 \%$ vs. $n=20 / 600,3.3 \%$; OR: 3.8 [95\% CI: 1.5-9.9]). This group of patients was also more likely to suffer from clinical sequelae at discharge $(n=15 / 46,35 \%$; vs. $\mathrm{n}=49 / 580,9.0 \%$; OR: 5.2 [95\% CI: 2.65-10.37]) with an increased absolute risk of requiring further ongoing care or rehabilitation ( $n=4 / 46,14 \%$ vs. $n=6 / 580,1.1 \%$; OR: 14.4 [95\% CI: 4.4-46.5]) and of recurrent Lemierre syndrome.

\section{Discussion}

Our recent analysis of cases of Lemierre syndrome reported in the past 20 years showed that this condition remains a dangerous disease which, even today, carries a significant risk of death and morbidity. ${ }^{2}$ The current analysis shows evidence of intracranial arterial or cardiac involvement in $7.7 \%$ of patients, which is higher than previous estimates. ${ }^{16}$ In addition, these patients had a much higher risk of death (12 vs. 3.3\%) and ongoing clinical sequelae (35 vs. 9\%), suggesting that such involvement may represent a marker of disease severity.

Previous studies have shown intracranial morbidity in up to $30 \%$ of cases of Lemierre syndrome. This included, along with stroke, manifestations such as meningitis, intracranial abscess, and sinus thrombosis. ${ }^{17}$ Although few case reports have described patients with arterial and cardiac events since the late 20th century, ${ }^{18}$ this is the first systematic analysis specifically looking at these complications of this disease, showing that Lemierre syndrome is a systemic disease that frequently involves the cerebral arterial district and the heart (excluding cases fulfilling the criteria for endocarditis).

The use of anticoagulation in Lemierre syndrome remains controversial. However, the high morbidity and mortality associated with arterial complications suggests that anticoagulation may play an important role. Our results show that patients with arterial involvement are already more likely to receive anticoagulation than those without; however, further research is required to assess whether anticoagulation can reduce the incidence of stroke, in particular in those with carotid involvement.

The high risk of death and complications suggests that neuroradiological and cardiac imaging may be considered already at baseline and if there is any relevant clinical deterioration. ${ }^{19}$ Computed tomographic (CT) scans of the head and neck including angiographic (CTA) and venographic (CTV) phases or magnetic resonance imaging (MRI; including MRA and MRV) can support physicians in assessing venous thrombotic extension, carotid arterial involvement, or intracranial sequelae. Doppler imaging of the jugular veins and carotid arteries may be a suitable alternative, as this patient population is relatively young and radiation exposure should be kept to a minimum. Ultrasound imaging remains the clinical standard to detect pericardial effusion, cardiac thrombi, and infective endocarditis. Special attention should be devoted to ruling out the presence of a right-to-left shunt, as venous embolization through a patent foramen ovale may contribute to the observed rate of arterial complications.

Stroke in patients with Lemierre syndrome may occur in several ways. These include spread from a septic or thrombotic embolus from arteritis or mycotic aneurysm, arterial vasospasm, passage of a venous embolus through a right-toleft shunt, as well as emboli from a pulmonary abscess or infective endocarditis.

The pathogenesis of arterial involvement of the carotid artery is likely from direct bacterial extension within the carotid sheath. Our results support this hypothesis, as patients with arterial involvement were also more likely to have severe adjacent venous disease. It has been proposed that the reason why we do not see arterial extension more commonly in Lemierre syndrome is a relative protective effect from the thicker wall of the artery in comparison to the adjacent vein. ${ }^{16}$

Our study has several limitations. First, data collected through systematic review are at risk of selection and publication bias. This is especially relevant for rare diseases and diseases for which no standardized, universally accepted definition exists. Our search and data collection strategy included several measures meant to limit this bias whenever possible (removal of language restriction, inclusion of the gray literature, snowballing, contact with individual study authors, and restriction to post-2000 cases to minimize heterogeneities in treatment). While this resulted in the largest dataset of cases of Lemierre syndrome described so far, because of the retrospective nature of the data, we cannot exclude potentially relevant residual bias. In particular, cases that did not satisfy the classic diagnostic criteria for Lemierre syndrome ("typical" Lemierre syndrome) may have been less likely to be correctly 
diagnosed and published, and studies using different criteria may result in different features of the population or observed incidence and distribution of the arterial complications. Second, while it is possible to specify the time of fist diagnosis of an arterial or cardiac complication, it cannot be established with certainty when the involvement actually occurred, and we cannot exclude that several may have already been present at diagnosis. Third, heterogeneity in treatment may remain despite the restriction to cases described over the past 20 years, because of the absence of agreed-upon guidelines on the management of this condition. ${ }^{20}$ In particular, treatment of arterial complications in a diverse population that includes pediatric patients and septic patients often in a critical care setting, a highly specialized field in constant evolution, may be especially relevant to confounding by indication or centerspecific expertise. Accordingly, no definitive conclusions on the efficacy or safety of alternative treatments can be drawn from these data. Rather, our findings provide a snapshot of current practice in the approach to a rare condition and may serve to generate hypotheses for future observational or interventional studies. ${ }^{20}$

In conclusion, Lemierre syndrome, a disease better known for its associated venous complications, carries a significant risk of arterial, intracranial, and cardiac involvement which are associated with a greater risk of long-term sequelae and death. Awareness of these lesser known complications is essential to enable timely diagnosis and help tailor management accordingly. The relatively high incidence of arterial involvement confirms the need for further research to assess the role of anticoagulation in Lemierre syndrome.

\section{What Is Known About This Topic?}

- To date there is a scarcity of published research regarding Lemierre syndrome despite the high morbidity and mortality associated with this disease which affects a predominantly young and previously healthy patient cohort.

- The venous thromboembolic phenomena are better known and have been more extensively documented; arterial and cardiac complications of Lemierre syndrome have also been sparsely reported.

\section{What Does This Paper Add?}

- In this study, we showed that Lemierre syndrome carries a significant risk of arterial, intracranial, and cardiac involvement.

- We also showed that cranial and cardiac complications are themselves associated with a greater risk of longterm sequelae and death.

Conflicts of Interest

Stefano Barco has received congress and travel payments from Daiichi-Sankyo and Bayer HealthCare; honoraria from Boston Scientific, Bayer HealthCare, and LeoPharma; and institutional research support from Sanofi, Concept Medical, Boston Scientific, and Bayer HealthCare. The other authors report no conflicts of interest.

\section{Acknowledgments}

Luca Valerio received the "Marco Brockhaus scientific award for Thrombosis Research in Paediatrics" from the Gesellschaft für Thrombose- und Hämostaseforschung (GTH) to promote and financially support research projects on the clinical presentation, treatment, and course of Lemierre syndrome and bacteria-associated venous thromboembolism in children and adolescents.

\section{References}

1 Valerio L, Pleming W, Pecci A, Barco S. Management of Lemierre syndrome. Minerva Med 2021;113(06):. Doi: 10.23736/S00264806.21.07497-8

2 Valerio L, Zane F, Sacco C, et al. Patients with Lemierre syndrome have a high risk of new thromboembolic complications, clinical sequelae and death: an analysis of 712 cases. J Intern Med 2021; 289(03):325-339

3 Batty A, Wren MW. Prevalence of Fusobacterium necrophorum and other upper respiratory tract pathogens isolated from throat swabs. Br J Biomed Sci 2005;62(02):66-70

4 Centor RM, Atkinson TP, Ratliff AE, et al. The clinical presentation of Fusobacterium-positive and streptococcal-positive pharyngitis in a university health clinic: a cross-sectional study. Ann Intern Med 2015;162(04):241-247

5 Brazier JS, Hall V, Yusuf E, Duerden BI. Fusobacterium necrophorum infections in England and Wales 1990-2000. J Med Microbiol 2002;51(03):269-272

6 Ramirez S, Hild TG, Rudolph CN, et al. Increased diagnosis of Lemierre syndrome and other Fusobacterium necrophorum infections at a Children's Hospital. Pediatrics 2003;112(05):e380

7 Bentham JR, Pollard AJ, Milford CA, Anslow P, Pike MG. Cerebral infarct and meningitis secondary to Lemierre's syndrome. Pediatr Neurol 2004;30(04):281-283

8 DeGaffe GH, Murphy JR, Butler IJ, Shelburne J, Heresi GP. Severe narrowing of left cavernous carotid artery associated with Fusobacterium necrophorum infection. Anaerobe 2013;22:118-120

9 Goyal MK, Kumar G, Burger R. Necrobacillosis resulting in isolated carotid thrombosis and massive stroke: a unique Lemierre variant? J Neurol Sci 2009;287(1-2):108-110

10 Root RW, Barrett TW, Abramo TJA. A 10-month-old with Lemierre syndrome complicated by purulent pericarditis. Am J Emerg Med 2013;31(01):274.e5-274.e7

11 Kreuzpointner R, Valerio L, Corsi G, et al. Ophthalmic complications of Lemierre syndrome. Acta Ophthalmol 2021 (epub ahead of print) . Doi: 10.1111/aos.14871

12 Abdel-Wahab N, Lopez-Olivo MA, Pinto-Patarroyo GP, SuarezAlmazor ME. Systematic review of case reports of antiphospholipid syndrome following infection. Lupus 2016;25(14): 1520-1531

13 George JN, Raskob GE, Shah SR, et al. Drug-induced thrombocytopenia: a systematic review of published case reports. Ann Intern Med 1998;129(11):886-890

14 Vazquez FJ, Paulin P, Rodriguez P, Lubertino M, Gándara E. The outcome of pulmonary vein thrombosis in non-surgical patients. A systematic review and case report. Thromb Haemost 2015;113 (05):1151-1154

15 Sacco C, Zane F, Granziera S, et al; Lemierre Study Group. Lemierre syndrome: clinical update and protocol for a systematic review and individual patient data meta-analysis. Hamostaseologie 2019;39(01):76-86 
16 Ratnasingham Y, Kristensen LH, Gammelgaard L, Balslev T. Arterial ischemic stroke as a complication to disseminated infection with Fusobacterium necrophorum. Neuropediatrics 2014;45(02): $120-122$

17 Wright WF, Shiner CN, Ribes JA. Lemierre syndrome. South Med J 2012;105(05):283-288

18 Jones TH, Bergvall V, Bradshaw JP. Carotid artery stenoses and thrombosis secondary to cavernous sinus thromboses in Fuso- bacterium necrophorum meningitis. Postgrad Med J 1990;66 (779):747-750

19 Touitou D, Deltour S, Bonneville F, et al. Hemiplegia in Lemierre syndrome: a neuroimaging strategy. Eur J Neurol 2006;13(04):424-426

20 Valerio L, Corsi G, Sebastian T, Barco S. Lemierre syndrome: current evidence and rationale of the Bacteria-Associated Thrombosis, Thrombophlebitis and LEmierre syndrome (BATTLE) registry. Thromb Res 2020;196:494-499 